\title{
Angles of Facial Harmony
}

\author{
Dr. Chohan Parvez Husain ${ }^{1}$, Dr. Mohammed Rizwan ${ }^{2}$, \\ Dr. Patil G. $\mathrm{S}^{3}$, Dr. Hemanth $\mathrm{M}^{4}$, Dr. Hareesha K. ${ }^{5}$ \\ Department of Orthodontics and Dentofacial Orthopaedics, Dayananda Sagar College of Dental Sciences, \\ Bangalore. Karnataka, India ${ }^{I}$. \\ Senior Lecturer, Department of Orthodontics and Dentofacial Orthopaedics,Dayananda Sagar College of \\ Dental Sciences, Bangalore.Karnataka, India 2 . \\ Professor and Head, Department of Orthodontics and Dentofacial Orthopaedics,Dayananda Sagar College of \\ Dental Sciences, Bangalore.Karnataka, India ${ }^{3}$. \\ Professor, Department of Orthodontics and Dentofacial Orthopaedics, Dayananda Sagar College of Dental \\ Sciences, Bangalore.Karnataka, India ${ }^{4}$. \\ Professor, Department of Orthodontics and Dentofacial Orthopaedics, Dayananda Sagar College of Dental \\ Sciences, Bangalore.Karnataka, India ${ }^{5}$.
}

\begin{abstract}
Objective: This study was undertaken to determine the total facial angle and subsequently the upper and lower facial angles to demonstrate various growth patterns by cephalometrics and to evaluate the validity of this method as compared with conventional cephalometrics.

Materials and methods: Lateral cephalograms of 100 individuals comprising of all males in the age range of 20 to 25 years seeking orthodontic treatment were taken. Lateral cephalograms of these individuals were analyzed by conventional cephalometrics and our new method to assess the growth pattern.

Results: The cut-off points of the Total Facial Angle were established as $61.30^{\circ} \pm 3.59^{\circ}$ for Horizontal Growth Pattern, $63.8^{\circ} \pm 2.99^{\circ}$ for Average Growth Pattern and $66.23^{\circ} \pm 3.02^{\circ}$ for Vertical Growth Pattern. Cut-off points for Upper and Lower Facial Angle were also established in all the three growth patterns. In Horizontal Growth Pattern, Upper Facial Angle was as $32.27^{\circ} \pm 1.74^{\circ}$ and Lower Facial Angle was $29.00^{\circ} \pm 2.15^{\circ}$. In Average Growth Pattern, Upper Facial Angle was $31.90^{\circ} \pm 1.49^{\circ}$ and Lower Facial Angle was $31.90^{\circ} \pm 1.49^{\circ}$. In Vertical Growth Pattern, Upper Facial Angle was $31.83^{\circ} \pm 1.56^{\circ}$ and Lower Facial Angle was $34.33^{\circ} \pm$ $1.42^{\circ}$.

Conclusion: From this study we were able to obtain a Total Facial Angle, the measurements of which were able to distinguish Horizontal, Average and Vertical Growth Patterns and proportion of Upper and Lower face was determined in the three growth patterns.

In Average Growth Pattern, the Upper and Lower Facial Angle were equal indicating a proportionate face. Whereas, in Horizontal Growth Pattern, Upper Facial Angle was greater than Lower Facial Angle and in Vertical Growth Pattern, Upper Facial Angle was lesser than Lower Facial Angle.
\end{abstract}

Keywords: Facial Harmony, Growth Pattern, Total Facial Angle, Upper Facial Angle, Lower Facial Angle.

\section{Introduction}

The primary role of conventional cephalometrics is evaluation of facial form through the use of angular and linear measurements. ${ }^{1}$ Majority of cephalometric analysis use methods that numerically compare a person's finding with average findings derived from so-called preselected 'standard' samples. ${ }^{2}$ The variability among the individuals becomes more complex because existing cephalometric analysis are based on chronological rather than maturational age and, thereby, ignore the individualized uniqueness of maturational development. ${ }^{2}$

Many investigators like Camper, ${ }^{3}$ Simon, ${ }^{4}$ Decoster, ${ }^{5}$ Moorees, ${ }^{6}$ Krogman, ${ }^{7}$ Sassouni, ${ }^{8}$ and Johnson ${ }^{9}$ presented different facial analyses that focus on a nonnumeric morphologic evaluation of the individual. Cephalometric analysis like Downs, ${ }^{10}$ Steiners, ${ }^{11}$ McNamara, ${ }^{12}$ Rakosi, ${ }^{13}$ are routinely used to evaluate facial growth pattern of an individual.

This new method demonstrates balance and disharmony in skeletal form based on the principle described by Archimedes.

\section{Aims and Objective}

2.1 To assess the reliability of the new method in determining growth patterns.

2.2 To establish cut-off points to distinguish Horizontal, Average and Vertical growth patterns, through Total Facial Angle.

2.3 To assess the upper and lower facial angles in horizontal, average and vertical growth patterns.

2.4 To compare the reliability of the new angles when compared to the existing cephalometric parameter. 


\section{Materials and Method}

This study was conducted with a total sample size of 100 individuals comprising of all males in the age range of 20 to 25 years, considering all had ceased growth and had expressed maximum growth potential, with no previous history of orthodontic treatment.

All digital lateral cephalograms were taken under standardized conditions at the Department of Radiology, Dayananda Sagar Dental College, once traced on a lead acetate paper with a $0.3 \mathrm{~mm}$ lead pencil, cephalometric analysis were performed using the conventional cephalometric parameters like Mandibular plane angle, ${ }^{10} \mathrm{Y}$ - axis, ${ }^{10}$ Go-Gn to S-N angle, ${ }^{11}$ Facial axis angle, ${ }^{12}$ Saddle angle, ${ }^{13}$ Articular angle, ${ }^{13}$ Gonial angle and Sum of angles, ${ }^{13}$ to determine their growth pattern. These cephalograms were categorized depending on the growth pattern as thirty horizontal growers, thirty average growers and thirty vertical growers depending on their cephalometric analysis; the other ten radiographs show technical errors and varied readings. ${ }^{14}$

The analyzed lateral cephalograms were subjected to a blind study by another examiner to employ the new method to assess the growth patterns. When both the groups were examined we could infer that in almost $78 \%$ of tracings the inferences of both the examiners were the same.

Therefore, the data obtained was tabulated and subjected through statistical test to check the reliability and to establish cut-off points to distinguish each growth pattern.

\subsection{Methodology - \\ Formation of the Angles of Harmony:}

3.1.1 Principles of orientation -

As described by Archimedes in $250 \mathrm{BC}$, if an equilateral triangle (ABC) is divided into halves it forms two adjacent triangles (ABD and $\mathrm{ACD}$ ) sharing two common sides (AD and $\mathrm{BC}$ ) forming angles 1 and 2 which are equal and in harmony (Figure 1 - Archimedes Principle). ${ }^{1}$

Applying this principle superficially to facial form, a triangle is constructed by joining the points Nasion, Basion and Gnathion that forms the Total facial angle (N-Ba-Gn) which is measured to evaluate the type of growth pattern. Further, this triangle is bisected by a line from Basion through point A to pass through the line Nasion Gnathion that forms the Upper face and Lower face represented by Upper facial angle (N-Ba-A) and Lower facial angle (A-Ba-Gn) which are measured to evaluate their contribution in determining the type of growth pattern (Figure 2 - Angles Of Facial Harmony).

\subsubsection{Construction of Angles of Harmony:}

1. Points used in the construction of angles are - Nasion (N), Basion (Ba), Gnathion (Gn) and point A.

2. Orientation Planes - Line N-Gn, Line N-Ba, Line Ba-A passing through point A and Line Ba-Gn.

3. Angles formed are-
a. Total Facial Angle (N-Ba-Gn)
b. Upper Facial Angle (N-Ba'-A)
c. Lower Facial Angle (A-Ba"-Gn)

3.1.3 The following measurements are recorded using a protractor (Figure 3 - Angular Measurements):

a. Total Facial Angle (N-Ba-Gn)

b. Upper Facial Angle (N-Ba-A)

c. Lower Facial Angle (A-Ba"-Gn).

The data obtained by tracing and analyzing the lateral cephalograms are tabulated and statistically analyzed to determine the Total Facial Angle in Horizontal Growth Pattern, Average Growth Pattern and Vertical Growth Pattern, to establish cut-off points to differentiate Horizontal Growth Pattern from Average Growth Pattern, Average Growth Pattern from Vertical Growth Pattern and to evaluate the Upper Facial Angle and Lower Facial Angle in all three growth pattern and infer their contribution in the development of the respective growth pattern.

\section{Results}

As per this new method cut-off points are derived for horizontal, average and vertical growth pattern. The cut-off points are as follows.

Total Facial Angle for Horizontal growth pattern is $61.30^{\circ} \pm 3.59^{\circ}$, for Average growth pattern is $63.8^{\circ} \pm 2.99^{\circ}$ and for Vertical growth pattern is $66.23^{\circ} \pm 3.02^{\circ}$ (Table I - Comparison of growth pattern in three respective groups).

For Horizontal growth pattern it was observed that the Upper Facial Angle is $32.27^{\circ} \pm 1.74^{\circ}$ and the Lower Facial Angle is $29.00^{\circ} \pm 2.15^{\circ}$ (Table I - Comparison of growth pattern in three respective groups).

For Average growth pattern it was observed that the Upper Facial Angle and the Lower Facial Angle were similar as $31.90^{\circ} \pm 1.49^{\circ}$ respectively. (Table I - Comparison of growth pattern in three respective groups). 
For Vertical growth pattern it was observed that the Upper Facial Angle is $31.83^{\circ} \pm 1.56^{\circ}$ and the Lower Facial Angle is $34.33^{\circ} \pm 1.42^{\circ}$ (Table I - Comparison of growth pattern in three respective groups).

The data when compared showed significant difference in total facial angle and lower facial angle in all the three growth patterns (Table II - Comparison of growth pattern difference in three respective groups).

Lateral cephalograms which were analyzed by the new method when compared to the previously traced and analyzed cephalograms using the existing parameters, i.e. Mandibular plane angle, ${ }^{10} \mathrm{Y}-$ axis, ${ }^{10}$ Go-Gn to S-N angle, ${ }^{11}$ Facial axis angle, ${ }^{12}$ Saddle angle, ${ }^{13}$ Articular angle, ${ }^{13}$ Gonial angle ${ }^{13}$ and Sum of angles ${ }^{13}$ indicated similar growth pattern validating that the new method is also reliable in ascertaining growth pattern accurately.

\section{Figures And Tables}

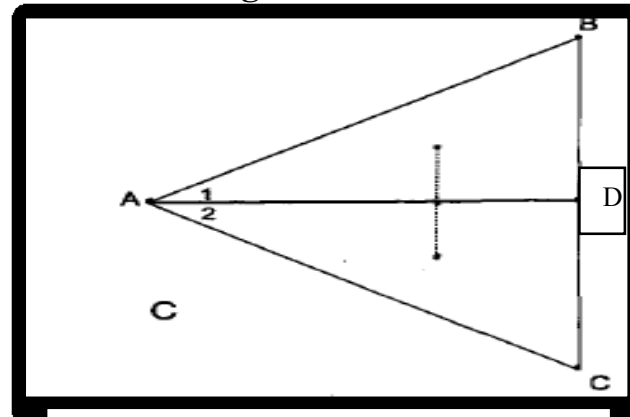

Figure 1 - Archimedes Principle

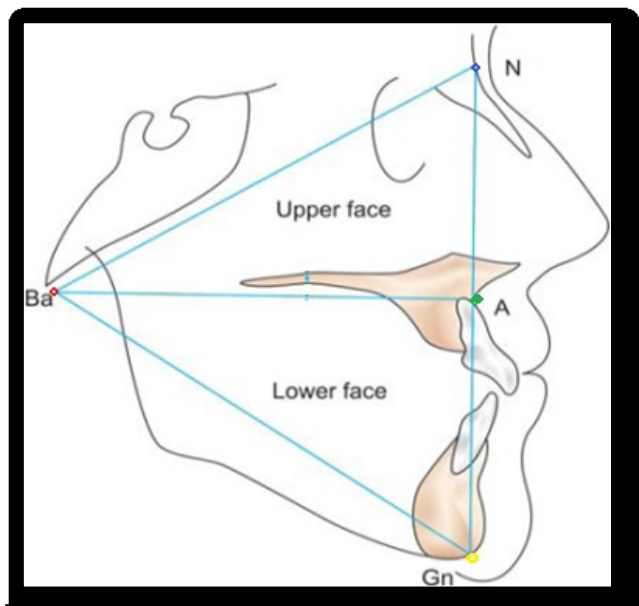

Figure 2 - Angles of Facial Harmony

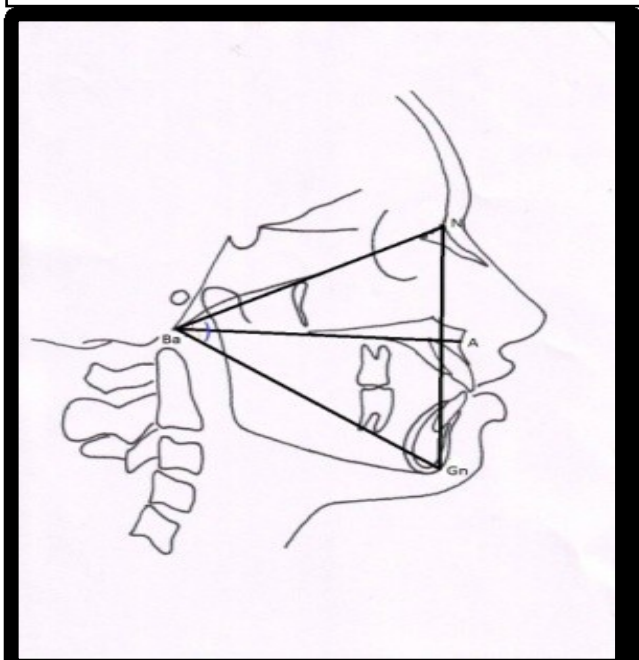

Figure 3 - Angular Measurements depicting Angle NBa'A and ABa"Gn. 
Table I: Comparison of growth pattern in three respective groups.

\begin{tabular}{|l|l|l|l|l|}
\hline Growth pattern & $\begin{array}{l}\text { Horizontal growth } \\
\text { pattern } \\
\text { (in degrees) }\end{array}$ & $\begin{array}{l}\text { Average growth } \\
\text { pattern } \\
\text { (in degrees) }\end{array}$ & $\begin{array}{l}\text { Vertical growth } \\
\text { pattern } \\
\text { (in degrees) }\end{array}$ & P value \\
\hline Total Facial Angle & $61.30 \pm 3.59$ & $63.8 \pm 2.99$ & $66.23 \pm 3.02$ & $<0.001^{* *}$ \\
\hline Upper Facial Angle & $32.27 \pm 1.74$ & $31.90 \pm 1.49$ & $31.83 \pm 1.56$ & 0.531 \\
\hline Lower Facial Angle & $29.00 \pm 2.15$ & $31.90 \pm 1.49$ & $34.33 \pm 1.42$ & $<0.001^{* *}$ \\
\hline
\end{tabular}

** Strongly significant $(\mathrm{P}$ value: $\mathrm{P} \leq 0.01)$.

Table II: Comparison of growth pattern difference in three respective groups.

\begin{tabular}{|c|l|l|l|}
\hline Growth pattern & $\begin{array}{c}\text { Horizontal growth pattern- } \\
\text { Average growth pattern } \\
\text { (in degres) }\end{array}$ & $\begin{array}{c}\text { Horizontal growth } \\
\text { pattern - Vertical } \\
\text { growth pattern } \\
\text { (in degrees) }\end{array}$ & $\begin{array}{c}\text { Average growth pattern- } \\
\text { Vertical growth pattern } \\
\text { (in degrees) }\end{array}$ \\
\hline Total Facial Angle & -2.500 & -4.933 & -2.433 \\
\hline$\bullet \quad$ Difference & $0.009^{* *}$ & $<0.001^{* *}$ & $0.012^{*}$ \\
\hline$\bullet \quad$ P value & & & 0.067 \\
\hline Upper Facial Angle $\quad$ Difference & 0.367 & 0.433 & 0.986 \\
\hline$\bullet \quad P$ value & 0.650 & 0.548 & \\
\hline Lower Facial Angle & & & -2.433 \\
\hline$\bullet \quad$ Difference & -2.900 & -5.333 & $<0.001^{* *}$ \\
\hline
\end{tabular}

* Moderately significant ( $\mathrm{P}$ value: $0.01<\mathrm{P} \leq 0.05$ ).

** Strongly significant (P value: $\mathrm{P} \leq 0.01$ ).

\section{Discussion}

Cephalometric analysis provides vital diagnostic information regarding dental and skeletal parameters. The present cephalometric study is designed to introduce a new cephalometric parameter to predict the growth patterns and to ascertain the contribution of the upper and the lower facial halves in the development of a certain growth pattern. Cephalograms help in identifying, locating and quantifying the nature of the problem.

Apart from diagnosis and evaluation of craniofacial morphology, cephalometrics help in developing a correct treatment plan for an individual. The various cephalometric parameters available for evaluating the growth pattern are Mandibular plane angle and Y - axis i.e. Downs Analysis, ${ }^{10}$ Go-Gn to S-N angle i.e. Steiner's analysis ${ }^{11}$ Facial axis angle i.e. McNamara Analysis, ${ }^{12}$ and Saddle angle, Articular angle, Gonial angle, Sum of angles i.e. Rakosi's and Jarabak Analysis. ${ }^{13}$

This new method was performed on the concept of Archimedes Principle which was superficially applied onto the facial form as it determines the facial growth pattern with simple landmarks and also adds light to the proportions of the face in the different growth patterns. This parameter proved reliable, convenient and easily applicable.

In this method, the points were selected on the basis of conventional cephalometric landmarks. Though Anterior Nasal Spine (ANS) is the most reliable landmark in the anterior mid face region, point A has been considered (even though it is an unreliable landmark) ${ }^{15}$ for this study on the basis that a line dropped perpendicular from Basion (Ba) when extended anteriorly on Nasion (Na) - Gnathion ( $\mathrm{Gn}$ ) line will intersect almost at Pt. A.

To check the reliability of this new method, pre-checked cephalometric samples were taken and cross examined. The new parameters were evaluated on the basis of this new method as total facial angle, upper facial angle and lower facial angle for horizontal, average and vertical growth patterns.

In this study the Total Facial Angle was established, to distinguish Horizontal from Average and Average from Vertical Growth Pattern wherein the cut-off point of Total Facial Angle was observed as $61.30^{\circ} \pm$ $3.59^{\circ}$ for Horizontal growth pattern, $63.8^{\circ} \pm 2.99^{\circ}$ for Average growth pattern and $66.23^{\circ} \pm 3.02^{\circ}$ for Vertical growth pattern. Further, the contribution of the Upper face and Lower face was evaluated in determining the type of growth pattern wherein the cut-off point for Upper Facial Angle as $32.27^{\circ} \pm 1.74^{\circ}$ and the Lower Facial Angle as $29.00^{\circ} \pm 2.15^{\circ}$ for Horizontal growth pattern, the Upper Facial Angle and the Lower Facial Angle 
being $31.90^{\circ} \pm 1.49^{\circ}$ for Average growth pattern, and Upper Facial Angle as $31.83^{\circ} \pm 1.56^{\circ}$ and the Lower Facial Angle as $34.33^{\circ} \pm 1.42^{\circ}$ for Vertical growth pattern was observed.

Thus an inference could be drawn that an Average growth pattern showed a well proportionate face where the Upper facial angle and Lower facial angle are equal. Whereas, in Horizontal growth pattern it was observed that the Upper facial angle was greater than the Lower facial angle and in Vertical growth pattern it was observed that the Upper facial angle was lesser than the Lower facial angle.

The new method applied to determine the growth patterns proved reliable, convenient and easy to perform. The cut-off points established for Total Facial Angle was able to distinguish Horizontal, Average and Vertical growth patterns. Further, the contribution of the Upper face and Lower face was determined in each of the three growth patterns through Upper and Lower facial angle. These new angles and parameters proved reliable as when compared with the existing cephalometric parameters, the same inference could be drawn.

\section{Conclusion}

The angular measurement which is here referred to as the 'Angles of Facial Harmony' is successful in distinguishing the three growth patterns.

Total facial angle was able to distinguish the Horizontal, Average and Vertical growth patterns.

Occurrence of a growth pattern is ascertained by comparing the Upper face to the Lower face in all three growth patterns.

Horizontal growth pattern showed upper facial angle greater than lower facial angle.

Average growth pattern showed upper facial angle equal to lower facial angle.

Vertical growth pattern showed upper facial angle lesser than lower facial angle.

All parameters used so far have No doubt been successful tools to predict the growth patterns. This method makes it even simpler to draw an inference on individual's growth pattern and it helps in determining the Facial Harmony as it assesses the facial form and evaluates the contribution of Upper face and Lower face in determining the growth patterns.

\section{References}

[1]. Fishman LA. Individualized evaluation of facial form. Am J Orthod Dentofacial Orthop 1997; 111:510-17.

[2]. Nehete AB, Hazare PV. An Evaluation of Centrographic Analysis as Compared with Conventional Cephalometric Analysis. J Ind Orthod Soc 2012;46(1):38-42

[3]. Moorrees AF. Twenty centuries of cephalometry. In: Alex Jacobson: Radiographic cephalometry from basics to videoimaging. Quintessence: 25 and 26.

[4]. Simon P. Fundamental principles of a systematic diagnosis of dental anomalies (transl BF Lischer) Boston: Stratford Company, 1926.

[5]. Decoster L. A network method of orthodontic diagnosis. Angle Orthod 1939;9:3-14.

[6]. Moores FA, Laure Lebret. The mesh diagram and cephalometrics. Angle Orthod 1962;32:214,31.

[7]. Krogman WM. The review of cephalometry. In: Krogman WM, Sassouni V (Eds). Syllabus in roentgenographic cephalometry.Philadelphia: College Offset; 1957:281.

[8]. Sassouni V. A reontgenographic cephalometric analysis of cephalofacial-dental relationships.Am J Orthod Dentofacial Orthop 1955;41:735-64.

[9]. Johnson JS. The use of centres of gravity in cephalometric analysis: A preliminary report. Dental Pract 1960;10:107-13.

[10]. Downs WB. Variations in facial relationship - Their significance in treatment and prognosis. Am J Orthod 1948; 34:812-823.

[11]. Steiner CC. Cephalometrics for you and me. Am J Orthod 1953; 39:729.

[12]. McNamara JA Jr. A Method of Cephalometric Evaluation. Am J Orthod. 1984:86:449-469.

[13]. Rakosi T. An atlas and manual of cephalometric radiography. London: Wolfe Medical Publication Ltd; $1982 ; 45-56$.

[14]. Mohammed Rizwan, Rohan Mascarenhas, Akhter Husain. Reliability of the existing Vertical Dysplasia Indicators in Assessing a Definitive Growth Pattern. Latin American Journal Of Orthodontics and Pediatric Dentistry, 2011.

[15]. Jacobson RL, Jacobson A. Point A revisited. 1980 Jan; 77(1):92-6. 